\title{
Mycobacterium tuberculosis of the elbow joint
}

\author{
Quintin Liao, ${ }^{1}$ James George Shepherd, ${ }^{2}$ Sulman Hasnie ${ }^{2}$
}

'Department of Orthopaedics, Queen Elizabeth University Hospital, Glasgow, UK ${ }^{2}$ Department of Microbiology, Glasgow Royal Infirmary, Glasgow, UK

\section{Correspondence to}

Dr Quintin Liao,

q.liao1@nhs.net

Accepted 27 November 2017

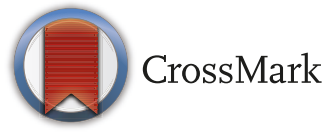

To cite: Liao Q, Shepherd JG, Hasnie S. BMJ Case Rep Published Online First: [please include Day Month Year]. doi:10.1136/bcr-2017 222735

\section{SUMMARY}

Mycobacterium tuberculosis(TB) affecting the elbow joint is rarely reported in the developed world. We present the case of an 85-year-old Caucasian female who complained of a chronically discharging and painful wound across her left elbow during her admission for an ischaemic stroke. This was initially deemed to be either a bursitis or local manifestation of amyloid by her general practitioner and dermatologist respectively prior to admission. She was commenced on flucloxacillin by the medical team for presumed cellulitis with minimal response. A synovial fluid sample and repeated wound swabs yielded no growth from routine bacterial culture. Radiological assessment together with knowledge of her husband having previous TB raised the possibility of TB arthritis. Synovial fluid aspirate was subsequently sent for acid-alcohol fast bacilli microscopy and mycobacterial culture that confirmed M. tuberculosis. She was consequently started on multidrug TB therapy, over a year after the onset of her symptoms.

\section{BACKGROUND}

Mycobacterium tuberculosis (TB) of the elbow joint is a rare condition occurring in 1\%-3\% of cases of skeletal TB. Skeletal TB occurs in approximately $2 \%$ of all TB cases reported. ${ }^{12}$ Early diagnosis and treatment is important to prevent osteoarticular destruction and preserve function. Diagnosis can be challenging owing to the non-specific and insidious nature of the clinical presentation. Furthermore, skeletal TB is often low on the initial differential list in patients presenting with joint pain, especially in areas of low endemicity and in the absence of constitutional or pulmonary symptoms. Correct diagnosis and treatment is therefore often delayed. We present a case of TB arthritis of the elbow joint that was subject to misdiagnosis and delayed treatment.

\section{CASE PRESENTATION}

An 85-year-old Caucasian female presented to hospital with right-sided arm weakness and expressive dysphasia. She was treated for a left-sided ischaemic stroke with antiplatelet therapy shortly after. During this admission, ongoing pain and discharge from a chronic wound across her left elbow was brought to the attention of the medical team. This was initially diagnosed as bursitis by her general practitioner (GP) 9 months prior to admission. Aspiration performed by the GP had revealed a raised white cell count (WCC) suggesting infection, but there were no bacteria seen on Gram stain microscopy and no growth from routine culture. The resultant wound from this procedure developed into an ulcerated lesion, and she was subsequently seen by dermatology as an outpatient. They adjudged it to be a new area of localised amyloid on a background of an existing amyloid lesion on her right lower limb. She was treated with low-dose corticosteroid with little effect prior to her acute admission.

Medical history was remarkable for localised amyloidosis on her right lower limb, cerebral aneurysm, hypertension, diverticulosis and mild cognitive impairment. It was noted that her husband had previous TB infection on admission; however, the significance of this information was not apparent until formal orthopaedic review.

\section{INVESTIGATIONS}

Examination demonstrated a swollen left elbow with two discharging sinuses over the olecranon wound as shown in figure 1. Active flexion was limited to $60^{\circ}$, and extension of the elbow was also limited owing to pain. Inflammatory markers on admission revealed a C reactive protein of $106 \mathrm{mg} / \mathrm{L}$ and WCC of $4.7 \times 10^{9} / \mathrm{L}$. The patient was initially commenced on flucloxacillin for presumed cellulitis, resulting in little clinical or biochemical improvement. Repeated wound swabs yielded no growth. Plain radiograph as shown in figure 2 revealed destruction of the olecranon and coronoid process of the ulna with extensive soft tissue swelling. Subsequent orthopaedic review suggested the possibility of tuberculous arthritis. Her husband's previous history of TB was noted and added to the existing clinical suspicion. On questioning, however, the patient denied any constitutional or respiratory symptoms. MRI was later performed as shown in figure 3 ; it revealed marrow oedema of the radius, ulna and humerus. Communications of the olecranon bursa and a separate volar collection were seen extending into the joint. Cutaneous sinus tract formation as well as a number of abnormal axillary nodes were also noted. She consequently underwent synovial fluid aspiration from the affected joint. Synovial biopsy for definitive tissue diagnosis was initially considered; however, the risks of poor wound healing with further formation of a chronic sinus was deemed too high. The specimen collected was sent to microbiology and pathology for further analysis. This was film positive for acidalcohol fast bacilli (AAFB) and culture positive for M. tuberculosis, which was fully sensitive to firstline antituberculous agents. Pathological analysis demonstrated granulomatous inflammation consistent with TB infection. Chest CT scan showed small bilateral pleural effusions, but no other pulmonary changes were seen. An HIV test was negative. 


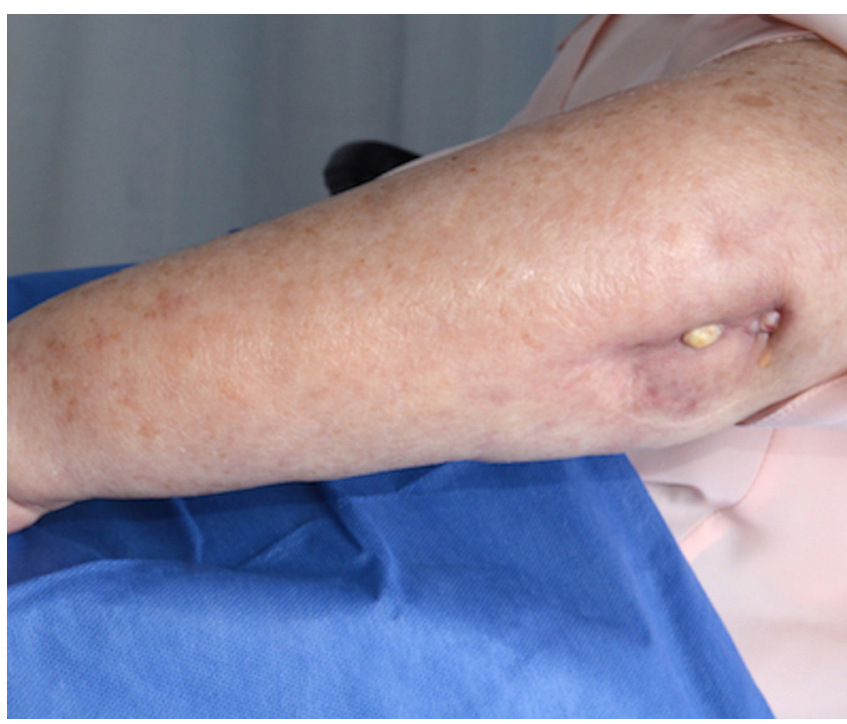

Figure 1 Picture of the chronic left elbow wound.

\section{DIFFERENTIAL DIAGNOSIS \\ - Trauma. \\ - Septic arthritis. \\ - Crystal arthritis. \\ - Inflammatory arthritis. \\ - Osteoarthritis. \\ - Haemophilic arthritis.}

\section{TREATMENT}

Surgical intervention was deemed to be inappropriate by the orthopaedic surgeons. She was started on antituberculous chemotherapy over a year after the onset of her symptoms. This consisted of an intensive phase of rifampicin, isoniazid, pyrazinamide and ethambutol for 2 months followed by a continuation phase of rifampicin and isoniazid for 4 months. She is currently in the process of finishing her continuation treatment.

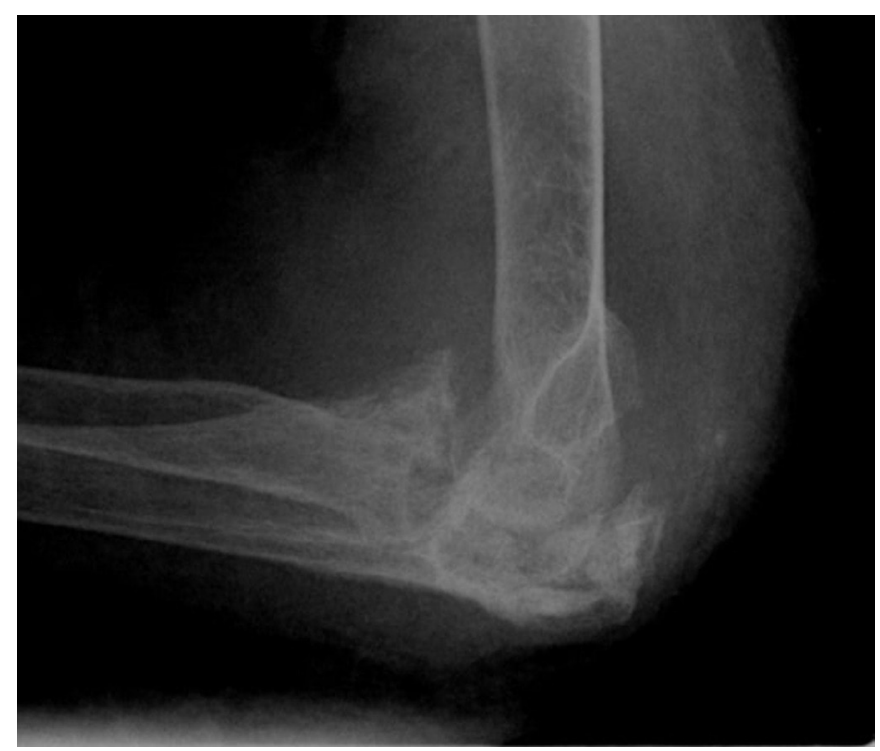

Figure 2 Plain radiograph of the left elbow showing destruction of the olecranon and coronoid process of the ulna with extensive soft tissue swelling.

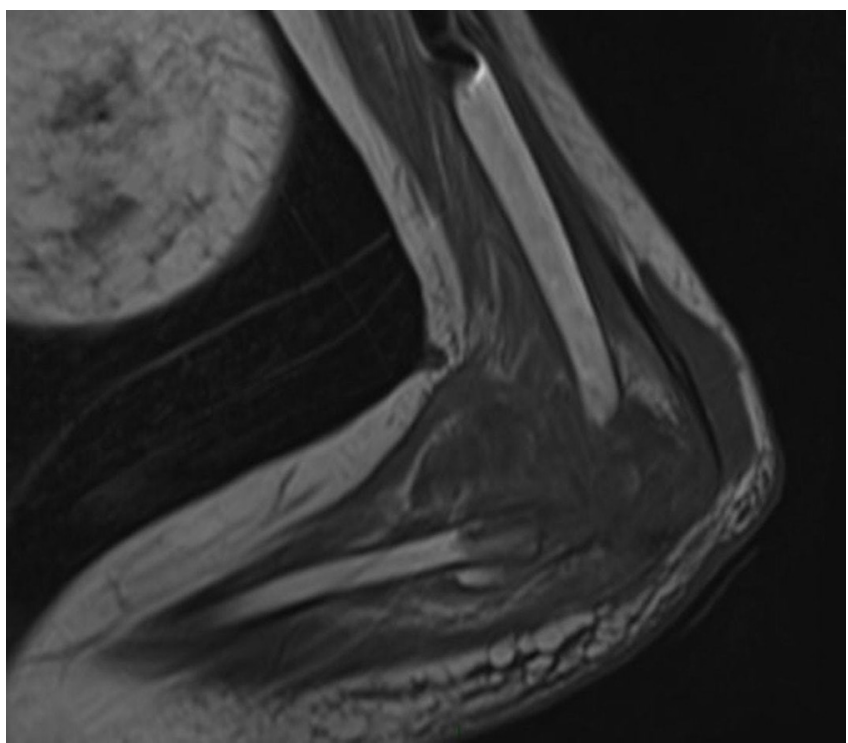

Figure $3 \mathrm{MRI}$ of the left elbow showing marrow oedema in the radius, ulna and humerus. $A 6 \times 3 \times 1.2 \mathrm{~cm}$ olecranon bursa and a $5 \mathrm{~cm}$ lobulated volar collection is seen communicating with the elbow joint. Cutaneous sinus tract formation is also seen.

\section{OUTCOME AND FOLLOW-UP}

The patient reports little clinical improvement in her range of motion and has required increasing doses of analgesia in the community to manage her pain. The wound continues to discharge through the sinus tracts and has yet to show signs of healing. This has required continuous dressings by the district nurse. Unfortunately, the patient has had several non-attendances in her follow-up appointments, and therefore adequate medication compliance could not be assessed.

\section{DISCUSSION}

Skeletal TB accounts for up to $35 \%$ of extrapulmonary manifestations of TB. ${ }^{3}$ It predominantly affects the spine (51\%), pelvis $(12 \%)$, hip and femur (10\%), the knee and tibia (10\%) and the ribs $(7 \%) .{ }^{4}$ Cases of affected non-weight-bearing joints such as the elbow are rarely reported in the medical literature.

Musculoskeletal TB can be a challenging diagnosis for the clinician. Clinically, it usually presents as local swelling and chronic pain of a single joint accompanied by gradual impairment in function. Cases of polyarticular TB are seen in 10\%-15\% cases. ${ }^{5}$

The classic systemic symptoms and pulmonary symptoms are frequently absent as this case has demonstrated. Furthermore, pulmonary features are seen in only half of patients undergoing a chest radiograph. ${ }^{6}$ Symptoms of skeletal TB are often vague and non-specific adding to the diagnostic challenge.

Our findings are similar to other reported cases in the literature. Madhavan et al described a comparable case of elbow joint TB infection, whereby constitutional and respiratory symptoms were also absent, with no evidence of radiographic chest features. Diagnosis was made on detection of AAFB and caseating granuloma on histology following negative routine gram stain and culture. ${ }^{7}$ Yazici et al also published a case of a chronically swollen and painful elbow joint, which was initially misdiagnosed as septic arthritis before ongoing symptoms prompted further investigation leading to the diagnosis and treatment for tuberculous arthritis. ${ }^{8}$ Although these cases are relatively rare, they highlight the diagnostic difficulties and resulting morbidity that can occur. 
Changes on plain radiograph of the affected joint are not always evident but can include a classical triad of features known as Phemister's triad: juxta-articular osteopaenia, joint space narrowing and osseous erosions. Other appearances include joint effusion and soft tissue swelling. ${ }^{9}$ MRI is a useful radiological tool to aid diagnosis. Features may include bone marrow changes, synovial thickening, joint effusion, abscess and sinus tract formation. ${ }^{10}$ Despite the role of imaging, these features are dependent on the stage of the disease progress and are non-diagnostic.

Definitive diagnosis requires aspiration or synovial biopsy. Microscopy and cultures of synovial fluid produce diagnosis in up to $80 \%$ of patients with skeletal TB. The remainder are diagnosed through synovial or bone biopsies with positive mycobacterial culture and identification of caseating granuloma on histopathological analysis. ${ }^{11}$

Due to the non-specific and insidious nature of skeletal TB, appropriate investigation and diagnosis is often delayed. The time from onset of symptoms to definitive diagnosis has been reported to range between 5 and 47 months. ${ }^{12}$ In this particular case, numerous samples from wound swabs and synovial fluid showed no organisms on microscopy and no growth on routine culture. Retrospectively, if skeletal TB was suspected at an earlier stage, samples could have been analysed for AAFB microscopy and mycobacterial culture that are not performed on routine bacterial samples.

The identification of known exposure and risk factors is important in allowing clinicians to suspect articular TB at an early stage. Risk factors include being in close contact with those with current or previous infectious TB. Immunosuppressive conditions such as HIV, diabetes and chronic kidney disease increase the risk for people to develop the active disease from the latent form. Thus, the screening of coinfectious diseases such as HIV is an important process in the management. Other risk factors include immunosuppressive medications, older age and poor nutrition. ${ }^{13}$ As in this case, the patient had documented exposure to her husband who had previous TB. This may have been transmitted before his TB therapy or during and after an incomplete course of treatment. Other risk factors included her older age and comorbidities leading to reduced immune function. This case highlights the importance in being able to recognise risk factors for skeletal $\mathrm{TB}$ as the diagnosis may have potentially been suspected at an earlier stage leading to earlier treatment.

Early diagnosis and treatment can be achieved through a combination of detailed history taking, clinical and radiological assessment together with a high index of suspicion for skeletal TB. This will help to prevent osteoarticular destruction and preserve maximal function of the joint.
Learning points

- It is important for clinicians to have a high index of suspicion for skeletal tuberculosis (TB), especially in patients with chronic joint pain not responding to conventional treatment.

- Specific testing for acid-alcohol fast bacilli on microscopy and mycobacterial culture should be requested in cases suspicious for skeletal TB.

- Clinicians should be able to identify risk factors and exposure risks for TB.

- Extrapulmonary TB is not always associated with constitutional symptoms or pulmonary involvement.

- Early diagnosis and treatment is important to maximise functional outcome by preventing osteoarticular destruction.

Contributors QL involved in drafting the manuscript and obtaining patient consent. JGS involved in providing information on microbiological analysis and also involved in editing of manuscript. SH involved in final editing.

Competing interests None declared.

Patient consent Obtained.

Provenance and peer review Not commissioned; externally peer reviewed.

(c) BMJ Publishing Group Ltd (unless otherwise stated in the text of the article) 2017. All rights reserved. No commercial use is permitted unless otherwise expressly granted.

\section{REFERENCES}

1 Tuli SM. Tuberculosis of the Skeletal System. 2nd edn. Delhi: Jaypee Brothers Medical Publishers, 1997.

2 Grosskopf I, Ben David A, Charach G, et al. Bone and joint tuberculosis-a 10-year review. Isr J Med Sci 1994:30:278-83.

3 Golden MP, Vikram HR. Extrapulmonary tuberculosis: an overview. Am Fam Physician 2005;72:1761-8

4 Aggarwal A, Dhammi I. Clinical and radiological presentation of tuberculosis of the elbow. ActaOrthop Belg 2006;72:282-7.

5 Kramer N, Rosenstein ED. Rheumatologic manifestations of tuberculosis. Bull Rheum Dis 1997:46:5-8.

6 Thimmaiah VT D. Unusual presentation of tuberculosis of elbow joint: a case report. RRJMHS 2013;2:17-20.

7 Madhavan A, Yagoub 0, Harrington P. Tuberculous infection of the elbow joint. European Journal of Orthopaedic Surgery \& Traumatology 2006;16:240-2.

8 Yazıcı A, Kayan G, Yaylacı S, et al. Tuberculous arthritis of the elbow joint: a case report. Eur J Rheumatol 2016;3:142-3.

9 De Backer Al, Vanhoenacker FM, Sanghvi DA. Imaging features of extraaxial musculoskeletal tuberculosis. Indian J Radiol Imaging 2009;19:176-86.

10 Hong SH, Kim SM, Ahn JM, et al. Tuberculous versus pyogenic arthritis: MR imaging evaluation. Radiology 2001;218:848-53.

11 Watts HG, Lifeso RM. Tuberculosis of bones and joints. J Bone Joint Surg Am 1996:78:288-98.

12 Hunfeld KP, Rittmeister M, Wichelhaus TA, et al. Two cases of chronic arthritis of the forearm due to Mycobacterium tuberculosis. Eur J Clin Microbiol Infect Dis 1998; 17:344-8.

13 Negin J, Abimbola S, Marais BJ. Tuberculosis among older adults-time to take notice. Int J Infect Dis 2015;32:135-7.

Copyright 2017 BMJ Publishing Group. All rights reserved. For permission to reuse any of this content visit

http://group.bmj.com/group/rights-licensing/permissions.

BMJ Case Report Fellows may re-use this article for personal use and teaching without any further permission.

Become a Fellow of BMJ Case Reports today and you can:

- Submit as many cases as you like

- Enjoy fast sympathetic peer review and rapid publication of accepted articles

- Access all the published articles

- Re-use any of the published material for personal use and teaching without further permission

For information on Institutional Fellowships contact consortiasales@bmjgroup.com

Visit casereports.bmj.com for more articles like this and to become a Fellow 